\title{
Radiation protection in Nuclear Medicine: a review on present situation in Sri Lanka
}

\author{
Manjula Hettiarachchi \\ Senior Lecturer in Nuclear Medicine, Faculty of Medicine, University of Ruhuna, Karapitiya, Galle.
}

Radiation is energy in the form of particles or waves. The term "radiation" is commonly used to refer to what scientists call "ionizing radiation," which is emitted from the nuclei of atoms. This is harmful to humans. Everyday, people are exposed to naturally occurring ionizing radiation from elements in the soil and air, cosmic rays, and even materials in the human body itself. Man-made devices such as $\mathrm{X}$-ray machines and nuclear power plants also generate radiation, although their output is tightly controlled to prevent harm. Some forms of radiation - including alpha and beta particles, are harmful only when they are ingested or come into contact with skin. This can be blocked by something as simple as a sheet of paper or a plate of glass. But "penetrating radiation"including gamma rays and neutron radiation produced in nuclear fission, could travel hundreds of yards through the air, penetrate normal walls or floors, and affect the entire human body ${ }^{1}$.

The Roentgen's discovery of X-ray (1895) and Becquerel's discovery of radioactivity (1896) were the important historical markers for Radiation Medicine. We remembered the $60^{\text {th }}$ anniversary of the Hiroshima and Nagasaki Abomb explosions, and passed the $20^{\text {th }}$ anniversary of Chernobyl accident. World political leaders are on heated arguments with Iran and North Korean involvement in nuclear power. On positive side, much credit is given to the remarkable medical diagnostic and therapeutic advances made using ionizing and non-ionizing radiations.

In the past two decade there has been a noticeable growth in Nuclear Medicine as well as Radiation Medicine in Sri Lanka. New units have been commissioned and the existing facilities have been up-graded.

\section{Regulatory system}

The Atomic Energy Regulatory Board was constituted under the Atomic Energy Act no 19 of 1969 by the Government of Sri Lanka ${ }^{2}$. The regulatory board draws its members from different task groups of various institutes involved in radiation work in some form. Under the provision of these regulations 'The Atomic Energy Authority (AEA)' was established under the Ministry of Science and Technology to oversee the scientific working in the field of radiation safety. Specific codes and standards of radiation safety on various applications of ionizing radiation are developed by the AEA and enforced to cover entire operational system from designing to ultimate functioning ${ }^{2}$.

\section{Regulation of radiation protection}

The atomic energy safety regulations no 1 of 1969 authorizes the AEA to adopt regulations, to license the users of radiation and transporters of radioactive sources and to conduct inspections to ensure radiation safety. The objective of the Regulatory Programme is the protection of general public, radiation workers and the environment from unwarranted exposure to ionizing radiation. AEA has the responsibility of implementing national regulations on Radiation Safety to achieve this objective. Atomic Energy Regulations adopted in 1975 were revised in $1999^{3}$. Revised regulations give the AEA the legal authority needed to implement a radiation protection programme that satisfies the requirements of the International Basic Safety Standards ${ }^{4}$.

\section{Authorization for medical practices}

An authorization for working with any radiation source(s) for medical applications such as human diagnosis and/or radiotherapy may be granted by the Authority upon fulfilling the 
requirements of Sections 12 to 13 of the regulations ${ }^{3}$, only to Medical practitioners, medical and health physicists, and to technologists in radiotherapy, radiology and Nuclear Medicine. They should fulfill the requirements of these regulations and possess the necessary knowledge of radiation protection related to work.

\section{Personnel Monitors and Protective Equipment}

Radiation workers are provided with suitable and adequate personal monitors and protective equipment. It may be:

1. personal dosimeters suitable for the type of radiation to be measured;

2. protective clothing;

3. protective respiratory equipment for the protection characteristics made known to the users; and

4. protective aprons and gloves and organ shields;

\section{Radiation Safety}

The principles followed in the radiation safety measurement ensure that workers and members of the public are not exposed beyond the specified limits as directed by the competent authority, follow the ALARA (As Low As Reasonably Achievable) principle, ensure safe handling and security of the radiopharmaceuticals, patient protection and waste management along with handling radiation emergencies. This is to ensure safety of persons handling radiation sources for medical applications, patients undergoing medical procedures, persons connected with the patient living with him and members of public unrelated to medical use of radiation. Aspects of radiation protection as relevant to the practice of Radiation/ Nuclear Medicine in Sri Lanka consider the following factors.

\section{Dose limitation}

The normal exposure of individuals shall be restricted so that neither the total effective dose nor the total equivalent dose to relevant organs or tissues, caused by the possible combination of exposure from practices, exceeds any relevant dose limit specified below, except in special circumstances at the discretion of the "Authority". Dose limits shall not apply to medical exposure from practices relating to radiation. No person shall unnecessarily expose himself, or others.

\section{Radiation worker}

a) Cumulative effective dose over a block of 5 yrs shall not exceed $100 \mathrm{mSv}$ ( $\mathrm{Sv}=$ Sieverts; defined as biologically effective dose (amount of ionization radiation required for absorption of 1 joule per kilogram of irradiated material) resulting from exposure to ionizing radiation.

b) Effective dose in any calendar year during the five years block shall not exceed $30 \mathrm{mSv}$.

c) Equivalent dose in any calendar year to the lens of the eye not exceed $150 \mathrm{mSv}$.

d) Effective dose in any calendar year to the skin, the hands and feet shall not exceed $500 \mathrm{mSv}$.

e) Special protection is given to the pregnant worker. Supplementary equivalent dose limit to the surface of the women's abdomen of $2 \mathrm{mSv}$ for the remainder of pregnancy.

\section{Trainees}

Effective dose in any calendar year shall not exceed $6 \mathrm{mSv}$.

\section{Public}

Effective dose in calendar year shall not exceed $1 \mathrm{mSv}$.

Radioactive waste management Principle of delay and decay is followed in waste management. For our convenience we have classified the radioactive waste into two categories;

a) Waste generated from diagnostic use.

b) Waste generated from therapeutic use. 
The waste generated from diagnostic use is mainly vials, syringes, needles and unused radioactivity. This is kept for decay for four weeks. Iodine is kept separately for three months. After that time the waste are monitored and moved in preparation for taken away. Packaging materials are always considered as radioactive waste, though it usually only needs to be monitored rather than stored. The exception to this is the iodine capsule can and capsule lead container, which are frequently contaminated. They are always stored for decay.

Precautions are maintained from patient's blood (in RIA lab), injected syringes and other work with blood products (i.e. HIV, Hepatitis B and C) in respective laboratory to avoid biohazard.

\section{Nuclear Medicine Units of Sri Lanka}

1. Nuclear Medicine Unit, Faculty of Medicine - Peradeniya.

2. Imaging unit, Department of Radiology National Hospital Colombo.

3. Nuclear Medicine Unit, Faculty of Medicine - Galle.

4. Imaging Unit, Department of Radiology Lady Ridgeway Children's Hospital, Colombo.

5. Radioimmunoassay (RIA) laboratory, Department of Pathology - National Hospital Colombo.

6. Nuclear Medicine Unit, Apollo Hospital, Colombo.

All the units mentioned above are now functioning with planar imaging except for NMU, Galle and RIA lab NHSL which have radioimmunoassay facility only. Imaging Unit of the LRH has a SPECT camera for renal imaging in paediatric patients. The two Medical Faculty units are strengthened by 4 Nuclear Medicine Physicians, 1 medical physicist and 8 technical officers. These units use ${ }^{125}$ I for RIA;

${ }^{131}$ I for thyroid uptake, whole body scan and for some thyroid scans and radio-iodine therapy; ${ }^{99} \mathrm{Tc}$ for other scans

Nuclear Medicine Unit of the Faculty of Medicine, University of Ruhuna provides invitro diagnostic service on several hormones and tumour markers. The next phase of development plan will focus on ${ }^{131} \mathrm{I}$ therapy unit followed by an imaging facility.

Following steps have been taken to ensure maximum safety:

1. Distribute the responsibilities among the occupational personal: Occupational personals (radiation safety officer (RSO), radiation safety supervisor (RSS), technical and other staff) are assigned with job descriptions and responsibilities along with radiation protection concept.

2. Classification of areas: Units classify three areas in view of reducing the risk of radiation hazard as ALARA by proper instructions on billboard, labeling (Caution! Radiation Area, Caution! Radioactive Material, Caution! Controlled Area, etc). General patients and the attendants are seated in separate areas from the injected patients (Supervised area); Only those who needed imaging and uptake are allowed to enter 'Controlled area' and no one is allowed to enter 'Restricted area' without proper authorization.

3. Immediate notification to the competent authority in case of any inadvertent spillage and contamination after ensuring all appropriate decontamination measures.

4. Radioactivity survey on all working areas could be conducted once a week and documented.

\section{References}

1. Liyanage C. Hettiarachchi M. Radionuclides in Biomedical Sciences. An Introduction. Foundation Books Pvt. Ltd. India. 2004.

2. Parliament of Ceylon, $4^{\text {th }}$ Session 1968-69. Atomic Energy Authority Act, No 19 of 1969.

3. Atomic Energy Authority. Regulations on ionizing radiation protection of the atomic energy safety regulations No.1 of 1999. Extracts of the Gazette No. $1142 / 30$ of July 2000.

4. IAEA Safety Standards Series-115. International Basic Safety Standards for Protection against Ionizing Radiation and for the Safety of Radiation Sources. Jointly prepared by IAEA, ILO, PAHO, and WHO. 1996. 